Supporting Information for

\title{
Aluminum Nanocrystals Grow into Distinct Branched Aluminum Nanowire Morphologies
}

Benjamin D. Clark, ${ }^{1,4}$ Minhan Lou, ${ }^{2,4}$ Peter Nordlander ${ }^{1,3,4}$ and Naomi J. Halas ${ }^{1,2,3,4}$

${ }^{1}$ Department of Chemistry, ${ }^{2}$ Department of Electrical and Computer Engineering, ${ }^{3}$ Department of Physics and Astronomy, ${ }^{4}$ Laboratory for Nanophotonics, Rice University, Houston, Texas 77005, United States

\section{Methods:}

\section{Al nanocrystal synthesis:}

All glassware, syringes and needles were dried at $120^{\circ} \mathrm{C}$ before use. All precursors were purchased from Sigma Aldrich and used as received unless otherwise noted. Dioctyl ether was dried over molecular sieves and sparged with argon before use. TIBA was procured as a $1.0 \mathrm{M}$ solution in hexanes. $50 \mathrm{mM}$ stock solutions of Tebbe's reagent were prepared in Schlenk flasks from 0.5 M Tebbe's reagent in anhydrous toluene in an argon filled glovebox with $<1 \mathrm{ppm}$ water and oxygen. For a typical reaction, a $100 \mathrm{~mL}$ three neck flask was secured to the Schlenk line with a reflux condenser and sealed with two rubber septa. All reactions were performed under an argon atmosphere using standard Schlenk techniques with fitted glass syringes and stainless steel needles. In a typical reaction, $0.2 \mathrm{~mL}$ of Tebbe's reagent $(50 \mathrm{mM})$ was added to $9 \mathrm{~mL}$ of dioctyl ether followed by the addition of $1 \mathrm{~mL}$ of TIBA $(1.0 \mathrm{M})$ at room temperature resulting in a solution with $100 \mathrm{mM}$ of TIBA and $1 \mathrm{mM}$ of Tebbe's reagent. The volume of Tebbe's reagent added was adjusted to produce Al NCs with different ratios of TIBA to Tebbe's reagent. The solution of Tebbe's reagent in dioctyl ether was orange and turned pale purple after the addition of TIBA. An inert high-temperature thermocouple (Omega TJ36-CASS-116G-12-OSTW-M) was inserted and the reaction solution was heated from room temperature to $\sim 200^{\circ} \mathrm{C}$ in $\sim 15$ minutes using a heating mantle controlled by an Omega CSC 32 series mini benchtop controller. While being heated the solution initially became colorless then golden brown followed by opaque brown and then black within $\sim 20$ minutes indicating the completion of the reaction. Alternatively an oil bath set to 200 ${ }^{\circ} \mathrm{C}$ was used but the reactions were found to take longer than 2 hours, likely due to a lower reaction temperature with this heating setup. Once complete, the reaction was cooled to room temperature 
and quenched with $10 \mathrm{~mL}$ anhydrous cyclohexane and transferred into a centrifuge tube. The sample was spun down at $5000 \mathrm{rcf}$ for 5 minutes and re-dispersed in $\sim 25 \mathrm{~mL}$ of isopropanol. The Al NPs were washed twice more at $12000 \mathrm{rcf}$ for 10 minutes and suspended in $25 \mathrm{~mL}$ of isopropanol for storage.

\section{TEM Imaging:}

TEM samples were prepared by drop casting a few $\mu \mathrm{L}$ of Al NPs in IPA onto the carbon-coated grid. Structural characterization of the Al nanocubes was performed by TEM with a JEOL 1230 operating at $80 \mathrm{kV}$. High-resolution TEM and selected area electron diffraction were performed using a JEOL 2100F TEM operating at $200 \mathrm{kV}$. Average Al NW diameter distributions were determined from measurements of TEM images using ImageJ software.

\section{UV-visible spectroscopy:}

A Cary $5000 \mathrm{UV}$-vis spectrometer was used to measure the extinction spectra of Al NW solutions in isopropanol using quartz cuvettes.

\section{FTIR spectroscopy:}

The Al NWs were transferred into tetrachloroethylene by centrifugation and removal of the isopropanol to prevent hydrocarbons from obscuring the Al NW signal in the IR. A Bruker Vertex $80 \mathrm{v}$ Fourier transform infrared (FTIR) spectrometer was used to measure the spectrum of branched Al NWs and Al NW bundles drop cast and dried onto $\mathrm{KBr}$ pellets from solutions in tetrachloroethylene.

\section{Electromagnetic simulations:}

Calculations of the optical properties of branched Al NWs of different sizes and morphologies in solution were performed by the finite-difference-time-domain (FDTD) method (Lumerical solutions 2019b). The NW cross section was pentagonal. The Al NW was coated with $4 \mathrm{~nm}$ thick alumina, thus the coated NW was modeled as a rounded Al pentagonal cylinder inside a rounded Alumina pentagonal cylinder. The radius of the Alumina pentagonal corner was $7 \mathrm{~nm}$ and that of the Al pentagon was $3 \mathrm{~nm}$. The edge length of the Alumina pentagon was $26 \mathrm{~nm}$ and that of the Al NW was $18 \mathrm{~nm}$ so that the width of the NW alumina pentagon was about $34 \mathrm{~nm}$ and 
that of the $\mathrm{Al}$ pentagon was about $26 \mathrm{~nm}$. The branched NWs were constructed by pentagonal cylinders accordingly. Since the resonance wavelength of long NWs were extending to the infrared, CRC aluminum, Palik alumina and constant 1.37 environment refractive indexes were used in all simulations. The unpolarized absorption and scattering cross sections were averaged through two orthogonal polarizations and six incoming directions $(+\mathrm{x},-\mathrm{x},+\mathrm{y},-\mathrm{y},+\mathrm{z},-\mathrm{z})$. A mesh size of $1 \mathrm{~nm}$ was used for all the Al NWs. The irradiation conditions for charge plots in Figure 4B are normal incident light propagation direction to the geometry plane and circular polarizations.

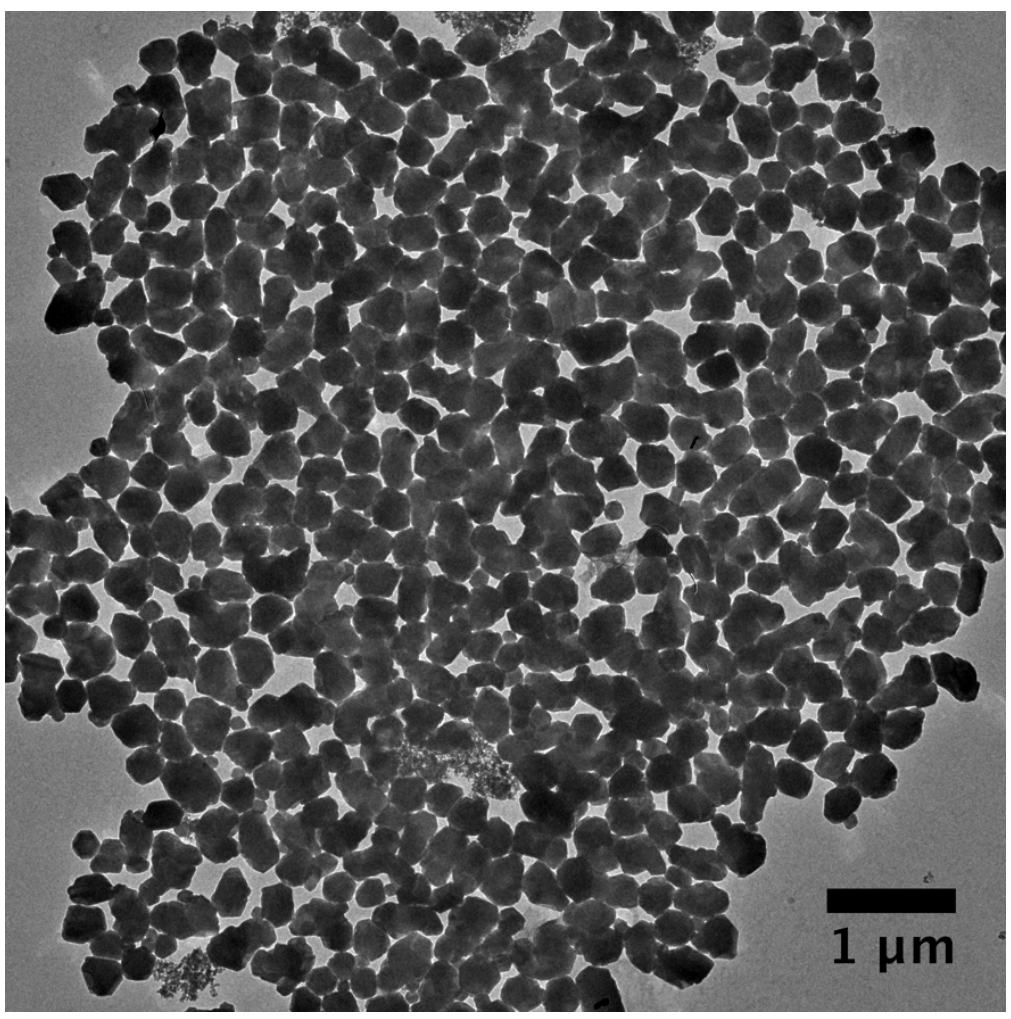

Figure S1: TEM of Al nanocrystals synthesized by titanium isopropoxide catalyzed thermal decomposition of TIBA at $200{ }^{\circ} \mathrm{C}$ in trioctylamine. 

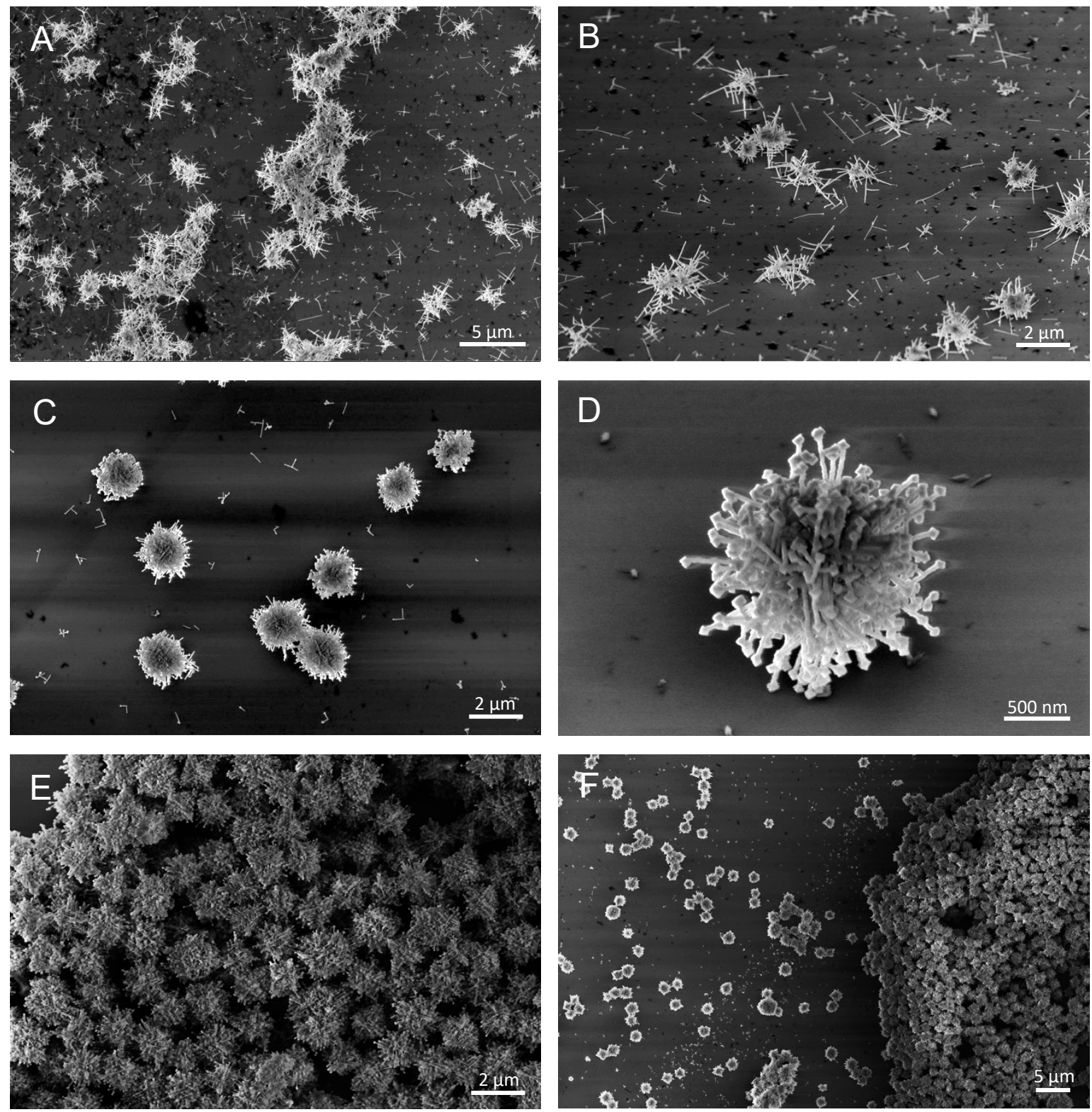

Figure S2: Representative SEM images of branched Al nanowires and Al nanowire bundles. A. branched Al nanowires from a 140:1 TIBA:Tebbe reaction. B. $45^{\circ}$ tilted image of branched Al nanowires from a 140:1 reaction. C. Al nanowire bundles from a 200:1 TIBA:Tebbe reaction. DF. Al nanowire bundles from a 300:1 TIBA:Tebbe reaction. The substrate in D is tilted by $45^{\circ}$ 

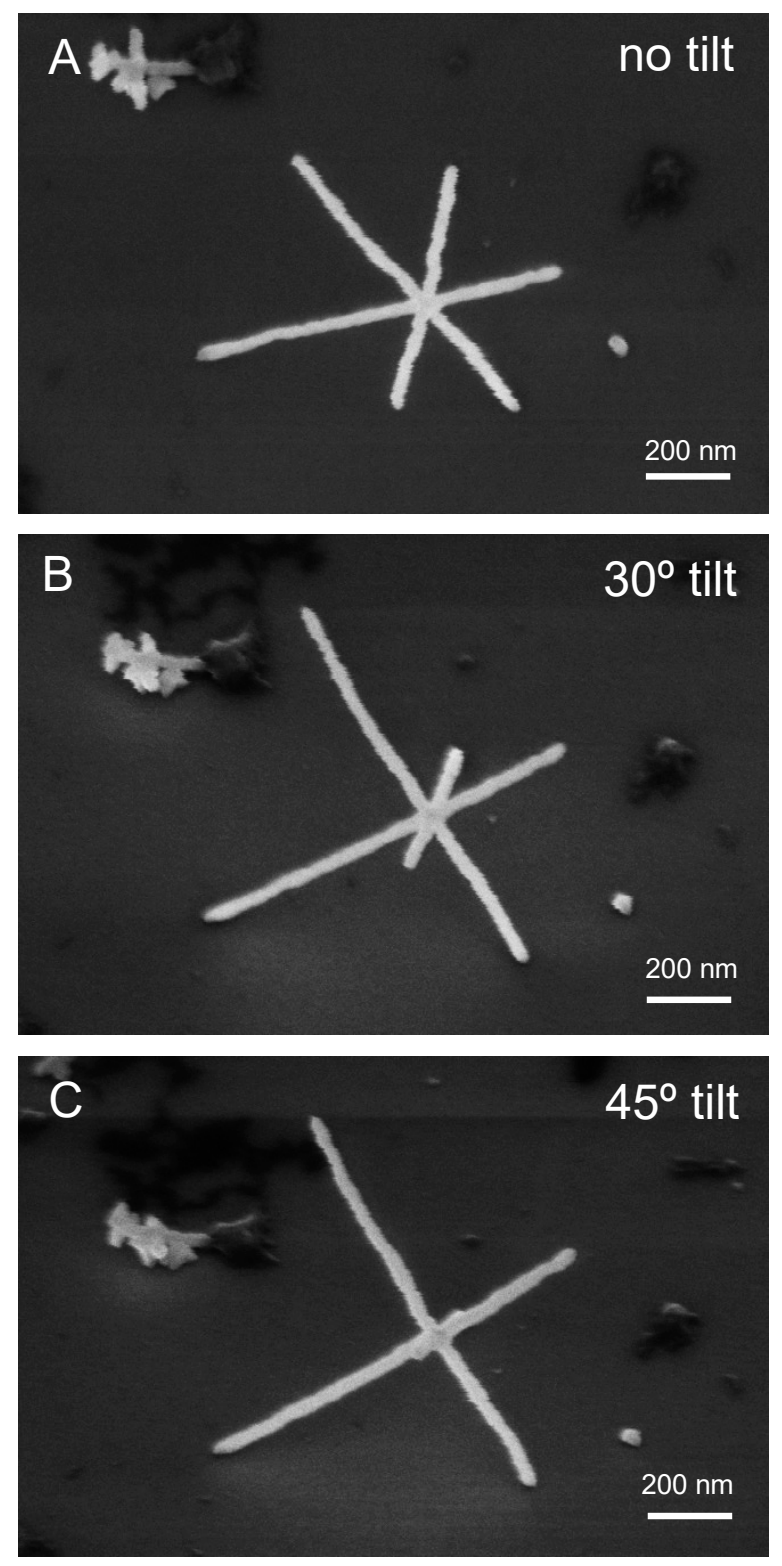

Figure S3: Series of tilted SEM images of a hexapod-shaped branched Al nanowire. A. No tilt. B. $30^{\circ}$ tilted substrate C. $45^{\circ}$ tilted substrate. 


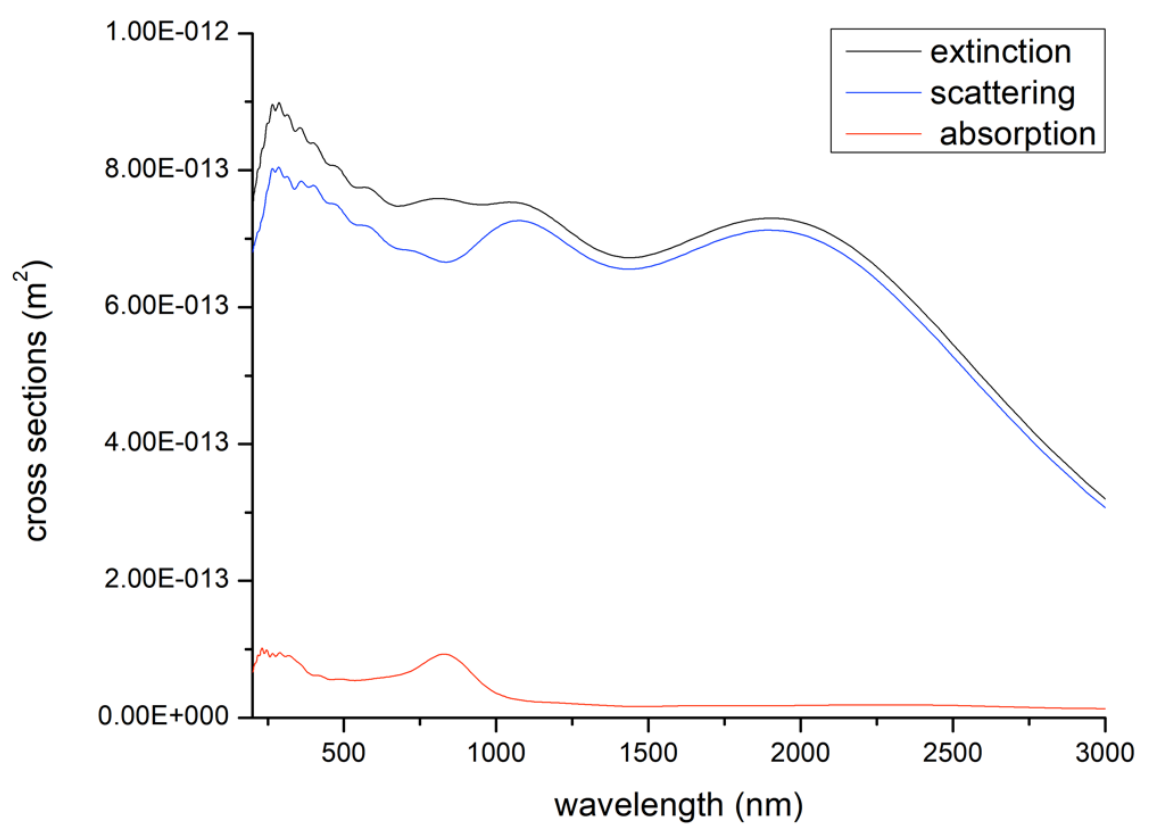

Figure S4: Extinction, scattering and absorption spectra of a $600 \mathrm{~nm} \mathrm{Al}$ sphere with a dipole plasmon mode at $\sim 2 \mathrm{um}$. 